INSECT IMMUNITY

\title{
Mechanism of adaptive immunity found in the fruitfly
}

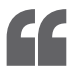

indicating that

vDNAs not

only allow for

amplification

of antiviral

responses but

also provide

immunological

memory
Although much has been learned from studying immune mechanisms in the fruitfly Drosophila melanogaster, there are fundamental differences between the invertebrate and vertebrate immune systems; in particular, the former is thought to rely solely on innate immunity for protection against pathogens. Now, a study in Cell by Andino and colleagues invites a rethink: they show that fruitflies can mount systemic adaptive responses against viruses, albeit with very different molecular and cellular mechanisms from those used by mammals.

Antiviral immunity in D. melanogaster is mediated by RNA interference (RNAi), a process whereby small interfering RNAs (siRNAs) guide an endonucleic RNA-induced silencing complex (RISC) to cognate viral transcripts. This leads to their cleavage by the RISC protein Argonaute-2 (AGO2) and thereby provides cell-autonomous specific immunity.

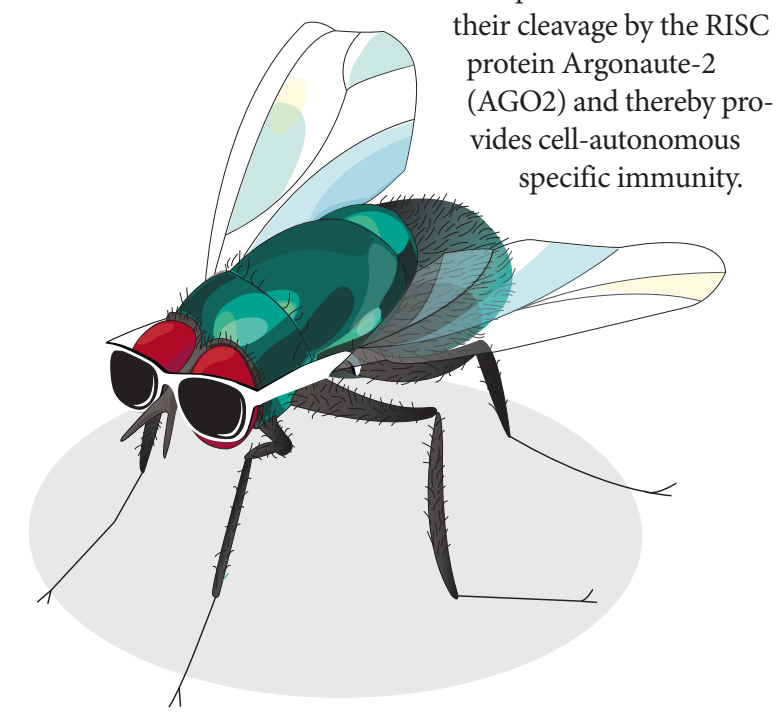

Whether RNAi signals from infected cells can spread systemically has been a matter of debate, as this would require as-yet-unidentified amplification and distribution steps.

To investigate potential systemic immune responses in D. melanogaster to infection with Sindbis virus (SINV), the authors focused on haemocytes, which are macrophage-like circulating immune cells that phagocytose virusinfected cells. They found that even though haemocytes were not directly infected by the virus, they appeared to confer protection, as flies in which haemocytes were genetically depleted had higher viral titres than wild-type flies. Interestingly, haemocyte-specific knockdown of AGO2 also led to higher viral titres, implying that these cells use an siRNA-based mechanism to limit infection.

To investigate whether this antiviral mechanism relies solely on primary virus-derived siRNAs (vsRNAs) acquired from phagocytosed infected cells, or whether it involves amplification of the vsRNAs, the authors developed an assay that distinguishes between primary vsRNAs, which carry a 5' monophosphate group, and secondary vsRNAs that are produced by de novo synthesis and have a $5^{\prime}$ triphosphate moiety. They found that in response to infection, haemocytes accumulate specific secondary vsRNAs in an AGO2dependent manner. Unlike plants and nematodes, which are known to amplify siRNAs by expressing RNA-dependent RNA polymerases, the primary vsRNAs were found to be reverse transcribed into virus-derived complementary DNAs (vDNAs), providing the template for secondary vsRNAs. Interestingly, haemocytes isolated 3 weeks - equivalent to one-third of the fly's lifetime - after infection still contained SINV-derived vDNAs, indicating that vDNAs not only allow for amplification of antiviral responses but also provide immunological memory.

These findings led to the question of how secondary vsRNAs in haemocytes can protect uninfected tissue. As naked siRNAs are labile and not efficiently taken up by other cells, the authors suspected that vsRNAs might be packaged into exosome-like vesicles (ELVs). Indeed, they found that flies with haemocytes that cannot produce ELVs have higher viral titres after SINV infection than wild-type flies. Moreover, ELVs isolated from the haemolymph of SINV-infected flies conferred virus-specific passive antiviral immunity when injected into naive flies.

The authors propose a model in which haemocytes acquire viral RNA by engulfing virus-infected cells. Reverse transcription into vDNA then allows for antiviral RNAi-mediated amplification and immune memory, and systemic protection is provided by secondary vsRNAs in ELVs, resulting in an adaptive immune mechanism that has striking parallels to mammalian adaptive immunity.

Alexandra Flemming

ORIGINAL ARTICLE Tassetto, M., Kunitomi, M. \& Andino, R. Circulating immune cells mediate a systemic RNAi-based adaptive antiviral response in Drosophila. Cell 169, 314-325.e13 (2017) 